\title{
81.
}

\section{ADDITION AU MÉMOIRE SUR QUELQUES TRANSMUTATIONS DES LIGNES COURBES.}

[From the Journal de Mathématiques Pures et Appliquées (Liouville), tom. xv. (1850), pp. $351-356$ : continued from t. xIV. p. $46,80$.

JE me propose de résumer ici la théorie des courbes du quatrième ordre, auxquelles donne lieu la première de mes méthodes de transmutation appliquée à une conique quelconque.

L'équation d'une telle courbe est de la forme -

$$
a x+b y+c z+2 f \sqrt{y z}+2 g \sqrt{z x}+2 h \sqrt{x y}=0,
$$

et nous avons déjà vu que cette courbe a pour tangentes doubles les trois droites $x=0, y=0, z=0$ (droites que nous avons représentées par $Q R, R P, P Q$ ). Pour trouver les autres propriétés de la courbe, mettons l'équation sous la forme

$$
\left(a-\frac{g h}{f}\right) x+\left(b-\frac{h f}{g}\right) y+\left(c-\frac{f g}{h}\right) z+\left(\sqrt{\frac{g h}{f}} \sqrt{x}+\sqrt{\frac{h f}{g}} \sqrt{y}+\sqrt{\frac{f g}{h}} \sqrt{z}\right)^{2}=0
$$

puis, en écrivant, pour plus de simplicité, $\frac{f x}{g h}, \frac{g y}{h f}, \frac{f z}{g h}$ au lieu de $x, y, z$, cette équation devient

$$
\left(\frac{a f}{g h}-1\right) x+\left(\frac{b g}{h f}-1\right) y+\left(\frac{c h}{f g}-1\right) z+(\sqrt{x}+\sqrt{y}+\sqrt{z})^{2}=0
$$

et de là, en mettant

$$
\left(\frac{a f}{g h}-1\right) x+\left(\frac{b g}{h f}-1\right) y+\left(\frac{c h}{f g}-1\right) z=-w
$$


l'équation de la courbe prend cette forme très simple

$$
\sqrt{x}+\sqrt{y}+\sqrt{z}+\sqrt{w}=0,
$$

en se souvenant toujours que les quantités $x, y, z, w$ satisfont à l'équation linéaire qui vient d'être donnée. Je représenterai dans la suite cette équation linéaire par

$$
\alpha x+\beta y+\gamma z+\delta w=0 .
$$

Il est évident que la droite $w=0$, de même que les droites $Q R, R P, P Q$, est tangente double de la courbe. De plus, ces quatre droites sont le système complet des tangentes doubles, car la courbe a, comme nous allons le voir, trois points doubles: en effet, la forme rationnelle de l'équation est

$$
\left(x^{2}+y^{2}+z^{2}+w^{2}-2 y z-2 z x-2 x y-2 x w-2 y w-2 z w\right)^{2}-64 x y z w=0,
$$

et, au moyen de l'identité

$$
(x+y)^{2}(z+w)^{2}-16 x y z w=(x-y)^{2}(z+w)^{2}+(x+y)^{2}(z-w)^{2}-(x-y)^{2}(z-w)^{2},
$$

cette équation rationnelle se transforme en

$$
\left[(x-y)^{2}-(z-w)^{2}\right]^{2}-4(x+y-z-w)\left[(x+y)(z-w)^{2}-(z+w)(x-y)^{2}\right]=0,
$$

laquelle fait voir que le point $(x=y, z=w)$ est un point double; de là aussi les points $(x=z, w=y),(x=w, y=z)$ sont des points doubles. Remarquons en passant qu'en supposant que les coefficients $\alpha, \beta, \gamma, \delta$ restent indéterminés, les droites $x=y$, $x=z, x=w$ seront des droites quelconques par les points $(x=0, y=0),(x=0, z=0)$, $(x=0, w=0)$ respectivement, et ces droites une fois connues, les droites $y=z, z=w$, $w=y$ seront déterminées, la première au moyen des points $(y=0, z=0),(y=x, z=x)$, la deuxième au moyen des points $(z=0, w=0),(z=x, w=x)$, et la troisième au moyen des points $(w=0, y=0),(w=x, y=x)$; et les trois droites ainsi déterminées se couperont nécessairement dans un même point. Cela revient au théorème suivant:

"Les trois points doubles d'une courbe du quatrième ordre avec trois points doubles sont les centres d'un quadrangle dont les côtés passent par les angles du quadrilatère formé par les tangentes doubles de la courbe."

Cette propriété des courbes du quatrième ordre dont il s'agit (je veux dire celle d'avoir trois points doubles) aurait dû faire partie du théorème général donné auparavant pour cette première méthode de transmutation.

En supposant que la conique à transmuter passe par le point $P$, on aura

$$
\alpha+\delta=0,
$$

et il suit de là que le point double $(x=w, y=w)$, identique dans ce cas avec le point $P$, se change en point de rebroussement, et en même temps que les droites $P Q$, $P R$ ne sont plus des tangentes doubles proprement dites, mais se réduisent à des 
tangentes simples qui passent par le point de rebroussement. Ajoutons que la tangente au point de rebroussement est la droite $x=w$.

En supposant que la conique à transmuter passe à la fois par les deux points $P$ et $Q$, nous aurons

$$
\alpha+\delta=0, \quad \beta+\delta=0
$$

Ici les deux points doubles $(x=w, y=z),(y=w, x=z)$, identiques dans ce cas avec les points $P, Q$, deviennent des points de rebroussement, les droites $Q R, R P, P Q$ ne sont plus des tangentes doubles proprement dites, mais les droites $R P, P Q$ sont les tangentes simples qui passent par les deux points de rebroussement respectivement, et la droite $P Q$ est la droite menée par les deux points de rebroussement. Ajoutons que les tangentes aux deux points de rebroussement respectivement sont les droites $x=w$ et $y=w$.

On sait qu'un cercle quelconque peut s'envisager comme conique qui passe par deux points fixes, savoir [les points circulainns à l'infini, c'est-à-dire] les points où l'infini, considéré comme droite, est rencontré par les deux droites imaginaires auxquelles se réduit un cercle évanouissant quelconque. En nommant ces droites les axes imaginaires de leur point d'intersection, prenons pour les droites $P Q, P R$ les axes imaginaires d'un point quelconque $P$, et pour la droite $Q R$ l'infini. Cela étant, un cercle quelconque sera transmuté dans une courbe du quatrième ordre ayant deux points de rebroussement aux points où l'infini est rencontré par les axes imaginaires du point $P$, ou, ce qui est la même chose, d'un point quelconque, et ayant de plus un point double. Et le point $P$, comme point d'intersection de deux axes imaginaires tangents de la courbe, est un foyer de la courbe (voyez le Mémoire de M. Plücker: Ueber solche Punkte die bei Curven höhern Ordnung cils der zweiten den Brennpunkten der Kegelschnitte entsprechen, Journal de M. Crelle, t. X. [1833] pp. 84-91). Cela suffit pour faire voir que la courbe est un limaçon de Pascal ayant le point $P$ pour le foyer qui n'est pas le point double. En effet, prenant pour vrai le théorème "Les ovales de Descartes ont deux points de rebroussement aux points où l'infini est rencontré par les axes imaginaires d'un point quelconque ${ }^{1,}$, comme cela revient à huit conditions, et qu'un ovale de Descartes peut être déterminé de manière à satisfaire à six conditions (ce qui fait en tout quatorze conditions, nombre des conditions qui déterminent une courbe du quatrième ordrẹ), toute courbe du quatrième ordre avec deux points de rebroussement, tels que nous venons de les mentionner, sera un ovale de Descartes, et si, de plus, la courbe du quatrième ordre a un point double, elle se réduira en limaçon (cas particulier, comme on sait, des ovales de Descartes). Donc, en résumé, tout cercle est transmuté dans un limaçon ayant un point fixe pour le foyer qui n'est pas le point double, théorème qui se rapporte à la méthode de M. Roberts pour le cas $n=\frac{1}{2}$. L'on doit cependant remarquer que cette méthode est due à M. Chasles. En effet, on trouve dans la Note citée de l'Aperçu historique, non-seulement la propriété des droites de se transmuter en des paraboles, mais aussi

1 M. Chasles a remarqué en passant (Note XXI. de l'Aperçu historique [Bruxelles, 1839]) que les ovales de Descartes ont deux points conjugués imaginaires à l'infini, théorème moins complet que celui que je viens d'énoncer. Pour la démonstration du théorème complet, voyez la Note à la suite de ce mémoire. 
celle des cercles de se transmuter en des ovales de Descartes (seulement M. Chasles paraît ne pas avoir remarqué que ces ovales étaient nécessairement des limaçons), et c'est la lecture de cette Note qui m'a appris cette théorie de la transmutation des cercles.

En supposant que la conique à transmuter passe par les trois points $P, Q, R$, nous aurons

$$
\alpha+\delta=0, \quad \beta+\delta=0, \quad \gamma+\delta=0
$$

les points doubles, identiques (dans ce cas) avec les points $P, Q, R$, deviennent des points de rebroussement, et les droites $Q R, R P, P Q$, au lieu d'être des tangentes doubles, sont tout simplement les droites qui. passent chacune par deux points de rebroussement. Ajoutons que les tangentes de la courbe, aux trois points de rebroussement respectivement, sont les droites $x-w=0, y-w=0, z-w=0$.

Il y a encore un cas particulier à considérer, savoir celui où la conique à transmuter est telle que, par rapport à cette conique, les points $Q$ et $R$ sont situés chacun dans la polaire de l'autre; on a alors $f=0$, cas qui échappe à l'analyse ci-devant employée. On voit sans peine que les deux points doubles $(y=w, x=z),(z=w, y=z)$ deviennent ici identiques, ce qui donne lieu à un point d'osculation. La droite $Q R$ et la droite $w=0$ ne sont plus des tangentes doubles proprement dites, mais ces droites deviennent l'une et l'autre identiques avec la tangente au point d'osculation.

Note sur les ovales de Descartes.

De l'équation de ces ovales,

$$
\sqrt{(x-a)^{2}+y^{2}}=m \sqrt{x^{2}+y^{2}}+n,
$$

on tire d'abord

$$
\left(1-m^{2}\right)\left(x^{2}+y^{2}\right)-2 a x+a^{2}-n^{2}=2 m n \sqrt{x^{2}+y^{2}},
$$

puis

$$
\begin{aligned}
&\left(1-m^{2}\right)^{2}\left(x^{2}+y^{2}\right)^{2}-4 a\left(1-m^{2}\right) x\left(x^{2}+y^{2}\right) \\
&+2\left[a^{2}\left(1-m^{2}\right)-n^{2}\left(1+m^{2}\right)\right]\left(x^{2}+y^{2}\right)+4 a^{2} x^{2}-4 a\left(a^{2}-n^{2}\right) x+\left(a^{2}-n^{2}\right)^{2}=0 .
\end{aligned}
$$

Pour trouver la nature de la courbe à l'infini, mettons $x+y i=\xi, x-y i=\eta, i=\sqrt{-1}$, et introduisons la quantité $\zeta$ de manière à rendre l'équation homogène. Cela donne

$$
\begin{aligned}
& \left(1-m^{2}\right)^{2} \xi^{2} \eta^{2}-2 a\left(1-m^{2}\right)(\xi+\eta) \xi \eta \zeta \\
& \quad+2\left[a^{2}\left(1-m^{2}\right)-n^{2}\left(1+m^{2}\right)\right] \xi \eta \zeta^{2}+a^{2}(\xi+\eta)^{2} \zeta^{2}-2 a\left(a^{2}-n^{2}\right)(\xi+\eta) \zeta^{3}+\left(a^{2}-n^{2}\right)^{2} \zeta^{4}=0
\end{aligned}
$$

ce qui fait voir, sans la moindre peine, qu'il y a des points de rebroussement aux points $(\xi=0, \zeta=0),(\eta=0, \zeta=0)$, savoir, aux points où l'infini, considéré comme droite, est rencontré par les droites $x+y i=0, x-y i=0$, qui sont les axes imaginaires $d u$ point $x=0, y=0$. 
Nous pouvons remarquer, en passant, que l'équation

$$
\left(1-m^{2}\right)\left(x^{2}+y^{2}\right)-2 a x+\left(a^{2}-n^{2}\right)=2 m n \sqrt{x^{2}+y^{2}},
$$

conduit, avec beaucoup de facilité, à une autre propriété, donnée par M. Chasles dans la Note déjà citée. En effet, en mettant

$$
a^{\prime}=\frac{n^{2}-a^{2}}{a\left(m^{2}-1\right)}, \quad m^{\prime}=\frac{m^{2}\left(n^{2}-1\right)}{a\left(m^{2}-1\right)}, \quad n^{\prime}=\frac{n}{a},
$$

cette équation se transforme en

$$
\left(1-m^{\prime 2}\right)\left(x^{2}+y^{2}\right)-2 a^{\prime} x+\left(a^{\prime 2}-n^{\prime 2}\right)=2 m^{\prime} n^{\prime} \sqrt{x^{2}+y^{2}},
$$

et par là on voit que l'équation primitive peut se transformer en

$$
\sqrt{\left(x-a^{\prime}\right)^{2}+y^{2}}=m^{\prime} \sqrt{x^{2}+y^{2}}+n^{\prime},
$$

c'est-à-dire, il y a toujours un troisième foyer de la courbe.

Il ne reste qu'à démontrer que la transformée (selon la méthode de M. Chasles) d'un cercle est toujours un limaçon. Soit, pour cela,

$$
r^{2}-2 a r \cos \theta+\delta=0
$$

l'équation du cercle; en mettant $\sqrt{m r}$ au lieu de $r$, et $\frac{1}{2} \theta$ au lieu de $\theta$, cette équation devient $m r-2 a \sqrt{m r} \cos \frac{1}{2} \theta+\delta=0$, ce qui donne $(m r+\delta)^{2}=2 a^{2} m r(1+\cos \theta)$, ou, en mettant $r \cos \theta=x$ et en réduisant à une forme rationnelle,

$$
\left[m^{2}\left(x^{2}+y^{2}\right)+\delta^{2}-2 a^{2} m x\right]^{2}-4 m^{2}\left(\bar{\delta}-a^{2}\right)\left(x^{2}+y^{2}\right)=0,
$$

ce qui appartient évidemment à un ovale de Descartes. En mettant $y=0$, l'équation devient

$$
\left[m^{2} x^{2}+2 m\left(\delta-2 \alpha^{2}\right) x+\delta^{4}\right](m x-\delta)^{2}=0,
$$

c'est-à-dire le point $m x-\delta=0, y=0$ est point double, ou la courbe est le limaçon de Pascal. 